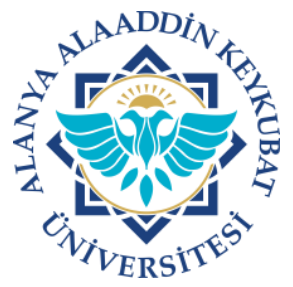

\title{
Burgers Benzeri Denklemin Bazı Seyahat Eden Dalga Çözümleri
}

\author{
İbrahim Enam İNAN' ${ }^{1}$, Doğan KAYA ${ }^{2}$ iD \\ ${ }^{1}$ Fırat Üniversitesi Ĕ̈itim Fakültesi, Matematik ve Fen Bilgisi Eğitimi Bölümü, İlköğretim Matematik \\ Öğretmenliği, Elazı̆g, Türkiye.einan@firat.edu.tr \\ ${ }^{2}$ İstanbul Ticaret Üniversitesi, Insan ve Toplum Bilimleri Fakültesi, Matematik Lisans Programı, \\ Istanbul,Türkiye.dogank@ticaret.edu.tr \\ Özet \\ Bu makalede, Burgers benzeri denklemin bazı seyahat eden dalga çözümlerini bulmak için Bäcklund Dönüşümü, \\ Benzerlik indirgeme ve Adomian Ayrıştırma yöntemleri denkleme uygulanmıştır. Yukarıdaki yöntemlerin denkleme \\ uygulanması sonucunda denklemin rasyonel, hiperbolik ve trigonometrik çözümleri elde edilmiştir. Daha sonra \\ Mathematica 11.2 programını kullanarak bu çözümlerin, denklemi sağladığı görülmüştür.
}

Anahtar Kelimeler: Burgers benzeri denklem, Bäcklund dönüşümü, Benzerlik indirgeme, Adomian Ayrı̧̧ım metodu.

\section{Some Travelling Wave Solutions of Burgers Like Equation}

\begin{abstract}
In this article, we have applied the Bäcklund Transformation, Similarity reduction and Adomian Decomposition methods to the equation to find some exact solutions of Burgers like equation. As a result of the application of abovementioned methods to the equation, we obtained the rational, hyperbolic and trigonometric solutions of the equation. Later, using Mathematica 11.2 program, we saw that these solutions satisfy the equation.
\end{abstract}

Keywords: Burgers like equation, Bäcklund transformation, Similarity reduction, Adomian Decomposition method.

\section{GIIRISŞ}

Lineer olmayan kısmi diferansiyel denklemler uygulamalı matematik ve fizikte önemli bir yere sahiptir. Bu denklemler ses, 1S1, difüzyon, elektrostatik, elektrodinamik, hidrodinamik, elastikiyet ve kuantum mekaniği gibi çeşitli kavramların modern bilimsel mantığının temellerini oluştururlar. Bu denklemleri çözmek için birçok analitik yöntem bulunmuştur [1-11]. Bulunan bu yöntemlerin yanı sıra lineer olmayan kısmi diferansiyel denklemleri çözmek için yardımcı bir denklem kullanarak çözüme ulaşabileceğimiz birçok metot vardır. $\mathrm{Bu}$ metotlar kullanılarak kısmi diferansiyel denklemler adi diferansiyel denklemlere dönüştürülür. Buradaki lineer olmayan kısmi diferansiyel denklemler, adi diferansiyel denklemler yardımıyla çözülür. Bu metotların bazıları [12-27] da verilmiştir. Ayrıca bu metotların ve bu metotlara benzer diğer pek çok metodun bazı lineer olmayan kısmi diferansiyel denklemlere uygulamaları [28-46] de verilmiştir. Bu çalışmada, Burgers benzeri denkleme; Bäcklund Dönüşümü, Benzerlik indirgeme ve Adomian Ayrıştırma metotları uygulanarak bu denklemin rasyonel, trigonometrik ve hiperbolik çözümleri elde edilmiştir. 


\section{METODLARIN ANALIZİ}

\subsection{HOMOJEN BALANS METODU}

Matematiksel fizikte bazı lineer olmayan kısmi diferansiyel denklemlerin özel çözümlerinin bulunmasında Homojen balans metodunun nasıl kullanıldığına bakalım. Bunun için iki değişkenli bir kısmi diferansiyel denklem

$$
\Psi\left(u, u_{x}, u_{t}, u_{x x}, u_{x t}, u_{t t}, \ldots\right)=0
$$

şeklinde verilmiş olsun. Burada, $\Psi$ bir polinom denklemi olup, polinomdaki alt simgeler ise kısmi türevleri göstermektedir. $w=w(x, t)$ fonksiyonunun (2.1.1) denkleminin yaklaşık çözümü olduğunu kabul edelim. Ancak, (2.1.2) deki fonksiyonların uygun bir lineer kombinasyonu şeklinde yazılan ve sadece bir değişkene bağlı olan $f=f(w)$ fonksiyonu (2.1.1) denkleminin bir gerçek çözümüdür. $f(w)$ dönüşümü kullanılarak (2.1.1) denklemi

$$
\Psi\left(1, f(w), f(w)_{x}, f(w)_{t}, f(w)_{x x}, f(w)_{x t}, f(w)_{t t}, \ldots\right)=0
$$

şeklinde yazılabilir. Burada $f(w)$ fonksiyonunun nasıl bulunduğunu $w=w(x, t)$ ve (2.1.2) deki fonksiyonların uygun bir lineer birleşimlerinin yaklaşık çözümlerinin nasıl oluşturulacağı tartış1lacaktır. Matematiksel fizikteki lineer olmayan bir denklemin özel çözümlerinin bulunmasında kullanılan bu metot, Homojen balans metodu olarak bilinir ve bu metot dört basamaktan oluşur.

(i) (2.1.2) denklemindeki fonksiyonların uygun bir lineer kombinasyonları seçilerek bunların katsayıları hesaplanır. Yani, verilen denklemdeki en yüksek mertebeden lineer olmayan terim ve en yüksek mertebeden lineer olan terim alınarak seçilen uygun bir lineer birleşim kullanılarak $f(w)$ fonksiyonunun gerekli türevleri ile beraber $w(x, t)$ fonksiyonunun kısmi türevlerindeki en yüksek eşitlikle beraber $f(w)$ ve $w(x, t)$ dönüşümleri kullanılarak (2.1.1) denklemi polinom şekline dönüştürülür. Burada oluşturulan en yüksek derecedeki eşitlikler çok önemlidir. Örneğin, verilen bir lineer olmayan kısmi diferansiyel denklemin en yüksek dereceden lineer olmayan terimi $u u_{x}$ ve en yüksek mertebeden lineer olan terimi $u_{x x x}$ olsun. (2.1.2) eşitliğindeki fonksiyonların lineer bir birleşiminin

$$
u=\frac{\partial^{m+n} f(w)}{\partial x^{m} \partial t^{n}}+f(w)
$$

eşitliğinin $(m+n)$. mertebeden daha düşük tüm kısmi türevli terimleri

$$
u=f^{(m+n)} w_{x}^{m} w_{t}^{n}+w(x, t)
$$

eşitliğindeki mevcut kısmi türevlerinin $(m+n)$. mertebesinden daha düşük tüm terimleri şeklinde olduğunu kabul edelim. Burada, $m \geq 0, n \geq 0$ olarak tanımlanan tamsayılardan oluşması gerekir. Bu durumda (2.1.3) eşitliği kullanılırsa,

$$
u u_{x}=f^{(m+n)} f^{(m+n+1)} w_{x}^{(2 m+1)} w_{t}^{2 n}+w(x, t)
$$

eşitliğinin mevcut kısmi türevlerinin $2(m+n)+1$ mertebesinden daha düşük tüm terimleri

$$
u_{x x x}=f^{(m+n+3)} w_{x}^{(m+3)} w_{t}^{n}+w(x, t)
$$


eşitliğinin mevcut kısmi türevlerinin $(m+n+3)$ mertebesinden daha düşük terimleri (2.1.4) ve (2.1.5) eşitliklerindeki $w(x, t)$ fonksiyonunun en yüksek mertebeden kısmi türevleri eşitlenirse, $2 m+1=m+3$ ve $2 n=n$ elde edilir. Buradan da $m=2$ ve $n=0$ bulunur. Bulunan bu $m$ ve $n$ değerleri (2.1.3) eşitliğinde kabul edilen lineer kombinasyonda yerine yazılırsa

$$
u=\frac{\partial^{2} f(w)}{\partial x^{2}}+f(w)
$$

şeklinde bir lineer birleşim seçilebilir. Yine benzer şekilde, verilen bir lineer olmayan kısmi diferansiyel denklemin en yüksek dereceden lineer olmayan terimi $u u_{x}$ ve en tüksek mertebeden lineer olan terimi $u_{x x}$ olsun. (2.1.2) ile verilen fonksiyonların lineer bir kombinasyonunun

$$
u=\frac{\partial^{m+n} f(w)}{\partial x^{m} \partial t^{n}}+f(w)
$$

eşitliğinin $(m+n)$. mertebeden daha düşük tüm kısmi türevli terimleri

$$
u=f^{(m+n)} w_{x}^{m} w_{t}^{n}+w(x, t)
$$

eşitliğinin mevcut kısmi türevlerinin $(m+n)$. mertebesinden daha düşük tüm terimleri şeklinde olduğunu kabul edelim. Burada, $m \geq 0, n \geq 0$ olan tamsayılardan oluşmalıdır. Bu durumda (2.1.7) eşitliği kullanılırsa,

$$
u u_{x}=f^{(m+n)} f^{(m+n+1)} w_{x}^{(2 m+1)} w_{t}^{2 n}+w(x, t)
$$

eşitliğinin mevcut kısmi türevlerinin $2(m+n)+1$ mertebesinden daha düşük tüm terimleri

$$
u_{x x}=f^{(m+n+2)} w_{x}^{(m+2)} w_{t}^{n}+w(x, t)
$$

eşitliğinin mevcut kısmi türevlerinin $(m+n+2)$ mertebesinden daha düşük terimleri (2.1.8) ve (2.1.9) eşitliklerindeki $w(x, t)$ nin en yüksek mertebeden kısmi türevleri eşitlenirse, $2 m+1=m+2$ ve $2 n=n$ elde edilir. Buradan da $m=1$ ve $n=0$ bulunur. Bulunan bu $m$ ve $n$ değerleri (2.1.7) eşitliğinde kabul edilen lineer birleşimde yerine yazılırsa

$$
u=\frac{\partial f(w)}{\partial x}+f(w)
$$

şeklinde bir lineer birleşim seçilebilir.

(ii) Birinci basamakta seçilen lineer birleşim (2.1.1) denkleminde yerine konularak, $w(x, t)$ nin en yüksek mertebeli türevleri ile bütün terimleri bir araya getirilerek sıfıra eşitlenir. Daha sonra $f(w)$ için bir adi diferansiyel denklem elde edilir ve bu denklem çözülür. Burada birçok durumda $f(w)$ bir logaritmik fonksiyondur.

(iii) Adi diferansiyel denklem ve onun yukarıda elde edilen çözümünden faydalanılarak, (ii) bendinde elde edilen ifadelerdeki $f(w)$ fonksiyonunun çeşitli türevlerine ait lineer olmayan terimler $f(w)$ nın daha yüksek mertebeli türevlerine dönüştürülür. Bu işlem yapıldıktan sonra, $f(w)$ fonksiyonunun aynı mertebeli türevleri bir araya getirilerek, $f(w)$ nın aynı mertebeli türevlerinin katsayıları sıfıra eşitlenir, bunun sonucunda $w(x, t)$ fonksiyonu için bir denklemler dizisi elde edilir. Bu denklemlerin sol tarafları $w(x, t)$ fonksiyonunun muhtelif türevlerinde $k$. dereceden homojen fonksiyonlardır. Buradaki $\mathrm{k}, f^{(k)} \mathrm{nın}$ mertebesidir. $\mathrm{Bu}$ denklemin homojen özellikleri göz önüne alınarak, $w(x, t)$ bazı sabitlerin bulunması gereken bir üstel fonksiyon olarak kabul edilir. Kabul edilen üstel fonksiyon $w(x, t)$, her bir $k$. dereceli 
homojen denkleminde yerine konarak bazı sabitlerin bulunması gereken bir dizi lineer olmayan cebirsel denklemler elde edilir. Bu lineer olmayan cebirsel denklemler için bir çözüm mevcutsa, o zaman $w(x, t)$ ve (i) bendinde seçilen lineer birleşimin katsayıları hesaplanır.

(iv) (i) bendinde seçilen lineer birleşim, (ii) ve (iii) bendinde hesaplanan $f(w)$ ve $w(x, t)$ yazılarak (2.1.1) denkleminin tam çözümü bulunmuş olur. Bu kesimin başında da belirtildiği gibi, açıklanan bu metodun iki uygulamasını aşağıda vererek ve bu iki uygulamayı kullanarak, bazı lineer olmayan kısmi diferansiyel denklemlerin çözümleri bulunacaktır [8].

\subsection{BÄCKLUND DÖNÜȘÜMÜ}

Metodun lineer olmayan bir kısmi diferansiyel denkleme nasıl uygulandığına bakalım. Verilen iki değişkenli bir kısmi diferansiyel denklem

$$
u_{t}=K\left(u, u_{x}, u_{x x, \ldots}\right)
$$

şeklinde olsun. Homojen balans metoduna göre (1) denkleminin Bäcklund dönüşümü

$$
u=\frac{\partial^{\alpha}}{\partial x^{\alpha}} f(w)+u_{0}
$$

olduğunu kabul edelim. Burada $f=f(w)$ ve $w=w(x, t)$ şeklindedir ve $u$ ile $u_{0}$ fonksiyonlarıda (2.2.1) denkleminin iki çözümüdür. $\alpha, u$ nun en yüksek mertebeden türevli lineer terimi ile $u$ fonksiyonunun en yüksek mertebeli lineer olmayan teriminin dengelenmesi ile tespit edilen bir sabittir. (2.2.2) dönüşümü (2.2.1) denkleminde yerine yazılarak elde edilen denklem en yüksek kuvvetten $w_{x}$ parantezine alınır, parantezin içinde $f$ fonksiyonuna bağlı bir adi diferansiyel denklem elde edilir ve bu denklem çözülerek $f(w)$ fonksiyonu bulunur. Bulunan bu $f(w)$ fonksiyonundan yararlanarak diğer bütün $f(w)$ ya bağlı olan türevler $f^{\prime}, f^{\prime \prime}, \ldots$ şeklinde ifade edilir ve son durumda denklem $f^{\prime}, f^{\prime \prime}, \ldots$ parantezlerine alınır. Daha sonra parantez içinde bulunan $w$ ya bağlı türevler birlikte yazılarak $w(x, t)$ hesaplanır. Hesaplanan $f(w)$ ve $w(x, t)$ fonksiyonları (2.2.2) dönüşümünde yerine yazılarak Bäcklund dönüşümü bulunur. Böylece, $\alpha$ ya bağlı olarak gerekli türevler alınır ve $u(x, t)$ çözüm fonksiyonu hesaplanmış olur. Bulunan bu $u(x, t)$ fonksiyonu (2.2.1) ile ele alınan lineer olmayan kısmi diferansiyel denklemin solitary dalga çözümüdür [9].

\subsection{BENZERLIKK INNDIRGEME}

Bu metodu açıklamak için genel lineer olmayan bir kısmi diferansiyel denklemi göz önüne alalım [8]. Bu iki değişkenli bir kısmi diferansiyel denklem

$$
u_{t}=K\left(u, u_{x}, u_{x x, \ldots}\right)
$$

şeklinde olsun. Homojen balans metoduna göre (2.3.1) denkleminin Benzerlik indirgemesinin

$$
u=\frac{\partial^{\alpha}}{\partial x^{\alpha}} f(w)+u_{0}
$$

şeklinde olduğunu kabul edelim, burada $\alpha, u$ fonksiyonunun en yüksek mertebeden türevli lineer terimi ile, $u$ fonksiyonunun en yüksek mertebeli lineer olmayan teriminin dengelenmesi ile tespit edilen bir sabittir. $\mathrm{Bu}$ yöntemde, (2.2.2) dönüşümünden farklı olarak $w$ ve $u_{0}$ fonksiyonlarını arıyoruz, burada $w=w(x, t)$ ve $u_{0}=u_{0}(x, t)$ şeklinde fonksiyonlardır. Daha sonra (2.3.2) dönüşümü (2.3.1) denkleminde yerine 
yazılarak en yüksek kuvvetten $w_{x}$ parantezine alınır, geriye kalan ifadelerde $f$ fonksiyonunun farklı türevlerinin parantezlerine alınarak, $f$ fonksiyonunun farklı türevlerinin katsayıları tespit edilir. Burada, $f$ fonksiyonunun farklı türevlerinin katsayıları oranı $w$ nın bir fonksiyonudur ve sadece $w$ için $f$ bir adi diferansiyel denklemdir. Daha sonra $w_{x}$ in en yüksek kuvvetinin katsayısı kullanılarak $w$ nın bir fonksiyonu olan $\Gamma$ nın belirlendiği her yerde, diğer katsayılar bulunurken $w_{x}^{n} \Gamma_{i}(w),(i=1,2, \ldots)$ oluşumundan yararlanılır. Burada, $\Gamma_{i}(w), w$ nın bazı keyfi fonksiyonlarıdır. Ayrıca, herhangi bir matematiksel ifade kullanıldığında ( diferansiyel, integral, logaritma alma, üstel, kuvvet alma, ..) bu ifadeler $\Gamma(w)$ ile belirlenir ve genelliği bozmaksızın bazı serbestlikler kullanılır. Bu serbestliklerin ne olduğunu uygulanan denklemlerde yeri geldiğinde verilecektir. Sonuç olarak bu adımlar takip edilerek verilen lineer olmayan kısmi diferansiyel denklemin rasyonel, trigonometrik ve hiperbolik olarak sırası ile ilerleyen çözümleri ile tek dalga çözümleri bulunmuş olacaktır [9].

\subsection{ADOMIAN AYRIŞIM METODU}

Adomian ayrışım metodunun bir seri metodu olduğu ve birçok cebirsel, lineer ve lineer olmayan diferansiyel denklemlere başarılı bir şekilde uygulandığı bilinmektedir. Metot genel olarak şu şekilde açıklanabilir. $F$, hem lineer hemde lineer olmayan terimleri içeren genel bir lineer olmayan adi diferansiyel operatör olmak üzere

$$
F u(x, t)=g(x, t)
$$

denklemi verilmiş olsun. (2.4.1) denkleminde $L$; verilen diferansiyel denklemin en yüksek mertebeden türevini, $R$; lineer operatörün kalan kısmını ve $N$; ise lineer olmayan terimi göstermek üzere (2.4.1) denklemini

$$
L u+R u+N u=g
$$

şeklinde ayrıştırarak yeniden yazalım. $L$ operatörünün tersi de mevcut olan bir lineer operatör olmak üzere, (2.4.2) eşitliği

$$
L u=g-R u-N u
$$

şeklinde yazılabilir. (2.4.3) ile verilen eşitliğin her iki tarafına $L$ operatörünün tersi olan $L^{-1}$ operatörü sol taraftan uygulanırsa

$$
L^{-1} L u=L^{-1} g-L^{-1} R u-L^{-1} N u
$$

eşitliği elde edilir. $L$ ikinci mertebeden ve tersi mevcut olan lineer bir operatör olarak Kabul edildiğinden, (2.4.4) eşitliğinde gerekli işlemler yapılırsa

$$
u(x, t)=u(x, 0)+L^{-1}(g(x, t))-L^{-1} R u-L^{-1} N u
$$

çözüm fonksiyonu bulunabilir. (2.4.5) ile elde edilen eşitlikteki $N u$ lineer olmayan terimleri

$$
N u=\sum_{n=0}^{\infty} A_{n}\left(u_{0}, u_{1}, \ldots, u_{n-1}\right)
$$

şeklinde ifade edilmektedir. Burada, $A_{n}$ polinomları özel Adomian polinomları olup bu polinomlar daha sonra incelenecektir. (2.4.5) eşitliğindeki $u(x, t)$, ayrıştırılmış seri çözüm fonksiyonudur. Bu seri çözüm fonksiyonunun birinci terimi $u_{0}$, verilen başlangıç şartı olan $u(x, 0)$ ve $L^{-1} g$ sağ taraf fonksiyonunun 
integrali olmak üzere $u_{0}=u(x, 0)+L^{-1} g$ ile bulunur. Daha sonra seri çözümün birinci terimi olan $u_{0}$ terimi kullanılarak serinin takip eden $u_{1}, u_{2}, \ldots$ terimleri elde edilerek Adomian ayrışımının seri çözüm fonksiyonu

$$
u(x, t)=\sum_{n=0}^{\infty} u_{n}(x, t)
$$

şeklinde elde edilir. Bu serinin yakınsak olduğu literatürde birçok çalışmada teorik olarak gösterilmiş̧tir. Bu seri çözümü kullanarak (2.4.5) eşitliği tekrar yazılacak olursa,

$$
\sum_{n=0}^{\infty} u_{n}=u_{0}-L^{-1} R \sum_{n=0}^{\infty} u_{n}-L^{-1} \sum_{n=0}^{\infty} A_{n}
$$

elde edilecek seri genel şekilde yazılabilir. Bu (2.4.7) eşitliği açık bir şekilde yazılacak olursa

$$
\begin{gathered}
u_{1}=-L^{-1} R u_{0}-L^{-1} A_{0}, \\
u_{2}=-L^{-1} R u_{1}-L^{-1} A_{1}, \\
u_{3}=-L^{-1} R u_{2}-L^{-1} A_{2}, \\
\vdots \\
u_{n+1}=-L^{-1} R u_{n}-L^{-1} A_{n}, n \geq 0
\end{gathered}
$$

eşitlikleri elde edilir. Buradaki $A_{n}$ polinomlarının her biri lineer olmayan terim için genelleştirilebilir. Yapılacak bu genelleştirmede $A_{0}$ sadece $u_{0}$ terimine, $A_{1}$ sadece $u_{0}$ ve $u_{1}$ terimlerine, $A_{2}$ ise $u_{0}, u_{1}, u_{2}$ terimlerine bağlı olmak üzere (2.4.8) eşitliğindeki bütün $A_{n}$ polinomları elde edilebilir. Genel olarak, $A_{n}$ Adomian polinomlarının bir kaç tanesi

$$
\begin{gathered}
A_{0}=f\left(u_{0}\right), \\
A_{1}=u_{1}\left(\frac{d}{d u_{0}}\right) f\left(u_{0}\right), \\
A_{2}=u_{2}\left(\frac{d}{d u_{0}}\right) f\left(u_{0}\right)+\left(\frac{u_{1}^{2}}{2 !}\right)\left(\frac{d^{2}}{d u_{0}^{2}}\right) f\left(u_{0}\right), \\
A_{3}=u_{3}\left(\frac{d}{d u_{0}}\right) f\left(u_{0}\right)+u_{1} u_{2}\left(\frac{d^{2}}{d u_{0}^{2}}\right) f\left(u_{0}\right)+\left(\frac{u_{1}^{3}}{3 !}\right)\left(\frac{d^{3}}{d u_{0}^{3}}\right) f\left(u_{0}\right), \\
\vdots
\end{gathered}
$$

şeklinde verilmektedir. Ayrışım polinomlarının en genel hali ise

$$
A_{n}=\frac{1}{n !}\left[\frac{d^{n}}{d \lambda^{n}} \Phi\left(\sum_{k=1}^{\infty} \lambda^{k} u_{k}\right)\right], n \geq 0
$$

şeklinde formüle edilmektedir [10].

3.ÖRNEK Burgers benzeri denklemi göz önüne alındığında,

$$
u_{t}+u_{x}+u u_{x}+\frac{1}{2} u_{x x}=0
$$

(3.1) ile verilen denklemin Homojen Balans metoduna göre

$$
u(x, t)=\frac{\partial^{\alpha}}{\partial x^{\alpha}} f(w)+u_{0}
$$


şeklinde Bäcklund dönüşümünü arayalım. Verilen denklemde, en yüksek dereceden lineer terim olan $u_{x x}$ ve en yüksek dereceden lineer olmayan terim $u u_{x}$ dengelenirse $\alpha=1$ bulunur ve (3.2) dönüşümü

veya

$$
u(x, t)=\frac{\partial}{\partial x} f(w)+u_{0}
$$

$$
u(x, t)=f^{\prime} w_{x}+u_{0}
$$

şeklinde olur. (3.4) dönüşümü (3.1) denkleminde yerine yazılır ve yeniden düzenlenirse,

$$
\begin{aligned}
\left(\frac{1}{2} f^{\prime \prime \prime}+f^{\prime} f^{\prime \prime}\right) & w_{x}^{3}+\left(f^{\prime \prime} w_{x} w_{t}+f^{\prime \prime} w_{x}^{2}+f^{\prime 2} w_{x} w_{x x}+f^{\prime \prime} w_{x}^{2} u_{0}+\frac{3}{2} f^{\prime \prime} w_{x} w_{x x}\right) \\
& +\left(f^{\prime} w_{x t}+f^{\prime} w_{x x}+f^{\prime} w_{x}\left(u_{0}\right)_{x}+f^{\prime} w_{x x} u_{0}+\frac{1}{2} f^{\prime} w_{x x x}\right)=0
\end{aligned}
$$

elde dilir ve (3.5) eşitliğinde $w_{x}^{3}$ ün katsayısı sıfıra eşitlenirse

$$
\frac{1}{2} f^{\prime \prime \prime}+f^{\prime} f^{\prime \prime}=0
$$

şeklinde bir lineer olmayan adi diferansiyel denklem elde edilir. Bu diferansiyel denklemden

$$
f=\ln w
$$

çözümü elde edilir. Bu çözümden

$$
f^{\prime 2}=-f^{\prime \prime}
$$

olarak bulunur. Bulunan bu ifade (3.5) eşitliğinde yerine yazılırsa son durumda,

$$
\left(w_{x} w_{t}+w_{x}^{2}+w_{x}^{2} u_{0}+\frac{1}{2} w_{x} w_{x x}\right) f^{\prime \prime}+\left(w_{x t}+w_{x x}+w_{x}\left(u_{0}\right)_{x}+w_{x x} u_{0}+\frac{1}{2} w_{x x x}\right) f^{\prime}=0
$$

elde edilir. Daha sonra $f^{\prime}$ ve $f^{\prime \prime}$ katsayılarını kullanarakda

$$
w_{x}\left(w_{t}+w_{x}+w_{x} u_{0}+\frac{1}{2} w_{x x}\right)+\frac{\partial}{\partial x}\left(w_{t}+w_{x}+w_{x} u_{0}+\frac{1}{2} w_{x x}\right)=0
$$

elde edilir. Buradan kolaylıkla görülebilir ki (3.8) denklemini

$$
w_{t}+w_{x}+w_{x} u_{0}+\frac{1}{2} w_{x x}=0
$$

sağlar. (3.4) ve (3.6) eşitliklerinden aradığımız Backlund dönüşümü

$$
u(x, t)=\frac{\partial}{\partial x} \ln w+u_{0}
$$

şeklinde elde edilir ve burada $w$, (3.9) denklemini sağlar. (3.1) denkleminin başlangıç çözümü $u_{0}=0$ alınırsa, bu durumda (3.9) ve (3.10) sirası ile 


$$
\begin{aligned}
& w_{t}+w_{x}+\frac{1}{2} w_{x x}=0 \\
& u(x, t)=\frac{\partial}{\partial x} \ln w
\end{aligned}
$$

şekline gelir. (3.11) denkleminin bir özel çözümü

$$
w(x, t)=1+\exp \left(x-\frac{3}{2} t\right)
$$

olarak alınırsa ve bu (3.12) eşitliğinde yerine yazıldığında (3.10) denkleminin solitary dalga çözümü

$$
u(x, t)=\frac{1}{2}+\frac{1}{2} \tanh \left(x-\frac{3}{2} t\right)
$$

olarak bulunur.

$\rightarrow$ (3.1) ile verilen denklemini tekrar göz önüne alıp, Benzerlik indirgeme metodu kullanılarak çözümünü arayalım. (3.1) denkleminin Benzerlik çözümünün (3.4) dönüşümünden

$$
u(x, t)=f^{\prime} w_{x}+u_{0}
$$

şeklinde olduğunu kabul edelim. (3.13) dönüşümü (3.1) denkleminde yerine yazılıp düzenlenirse

$$
\begin{aligned}
\left(\frac{1}{2} f^{\prime \prime \prime}+f^{\prime} f^{\prime \prime}\right) & w_{x}^{3}+\left(f^{\prime \prime} w_{x} w_{t}+f^{\prime \prime} w_{x}^{2}+f^{\prime 2} w_{x} w_{x x}+f^{\prime \prime} w_{x}^{2} u_{0}+\frac{3}{2} f^{\prime \prime} w_{x} w_{x x}\right) \\
& +\left(f^{\prime} w_{x t}+f^{\prime} w_{x x}+f^{\prime} w_{x}\left(u_{0}\right)_{x}+f^{\prime} w_{x x} u_{0}+\frac{1}{2} f^{\prime} w_{x x x}\right) \\
& +\left(\left(u_{0}\right)_{t}+\left(u_{0}\right)_{x}+u_{0}\left(u_{0}\right)_{x}+\frac{1}{2}\left(u_{0}\right)_{x x}\right)=0
\end{aligned}
$$

elde edilir. (3.14) eşitliği $f^{\prime}, f^{\prime \prime}$ ve $f^{\prime 2}$ parantezlerine alınırsa bu eşitlik

$$
\begin{gathered}
\left(\frac{1}{2} f^{\prime \prime \prime}+f^{\prime} f^{\prime \prime}\right) w_{x}^{3}+w_{x} w_{x x}{f^{\prime}}^{2}+\left(w_{x} w_{t}+w_{x}^{2}+w_{x}^{2} u_{0}+\frac{3}{2} w_{x} w_{x x}\right) f^{\prime \prime}+\left(w_{x t}+w_{x x}+\right. \\
\left.w_{x}\left(u_{0}\right)_{x}+f^{\prime} w_{x x} u_{0}+\frac{1}{2} w_{x x x}\right) f^{\prime}+\left(\left(u_{0}\right)_{t}+\left(u_{0}\right)_{x}+u_{0}\left(u_{0}\right)_{x}+\frac{1}{2}\left(u_{0}\right)_{x x}\right)=0
\end{gathered}
$$

şeklinde yazılabilir. Bir önceki örnekteki açıklamalar göz önüne alınırsa

$$
\begin{aligned}
& w_{x} w_{x x}=w_{x}^{3} \Gamma_{1}(w) \\
& w_{x} w_{t}+w_{x}^{2}+w_{x}^{2} u_{0}+\frac{3}{2} w_{x} w_{x x}=w_{x}^{3} \Gamma_{2}(w) \\
& w_{x t}=w_{x}^{3} \Gamma_{3}(w) \\
& w_{x x}+w_{x}\left(u_{0}\right)_{x}+f^{\prime} w_{x x} u_{0}+\frac{1}{2} w_{x x x}=w_{x}^{3} \Gamma_{4}(w) \\
& \left(u_{0}\right)_{t}+\left(u_{0}\right)_{x}+u_{0}\left(u_{0}\right)_{x}+\frac{1}{2}\left(u_{0}\right)_{x x}=w_{x}^{3} \Gamma_{5}(w),
\end{aligned}
$$

burada $\Gamma_{i}(i=1,2, \ldots, 5) \mathrm{w}$ nın belirlenecek bazı keyfi fonksiyonlarıdır. $u_{0}$ ve $w$ nın belirlenmesinde genelliği bozmaksızın aşağıdaki kuralların kullanılabileceği iki serbestlik vardır:

(a) $u_{0}=u^{\prime}(x, t)+\frac{\partial}{\partial x} \Omega$, şeklindeyse o zaman, $\Omega=0$ alınabilir. $(f(w) \rightarrow f(w)-\Omega$ dönüşümü yapılarak). 
(b) $w(x, t), \quad \Omega(w)=w_{0}(x, t)$ şeklindeki bir denklem ile tanımlanmış ise $\Omega=w$ olarak alınabilir. ( $w \rightarrow$ $\Omega^{-1}(w)$ dönüşümü yaparak).

(a) ve (b) serbestlikleri kullanılarak (3.16) - (3.20) denklemlerinin genel çözümleri bulunabilir. Şöyle ki (3.16) ve (3.17) eşitlikleri alınır ve yukarıda verilen (a) ve (b) serbestlikleri kullanılırsa,

$$
w=x \theta(t)+\sigma(t)
$$

olarak hesaplanır. (3.17) ve (3.21) eşitlikleri kullanılarak

$$
w_{x}=\theta(t), w_{x x}=0, w_{x}^{2}=\theta^{2}(t), w_{t}=x \frac{d \theta}{d t}+\frac{d \sigma}{d t}
$$

bağıntıları bulunur. Bunlar (3.17) de yerlerine yazılırsa,

$$
u_{0}=-\frac{1}{\theta}\left(x \frac{d \theta}{d t}+\frac{d \sigma}{d t}\right)-1
$$

elde edilir. Elde edilen (3.21) ve (3.22) bağıntıları (3.16) - (3.20) denklemlerinde kullanılırsa

$$
\begin{gathered}
\Gamma_{1}=\Gamma_{2}=0, \Gamma_{3}=A, \Gamma_{4}=-A, \Gamma_{5}=-A^{2} w-B \\
\sigma^{\prime \prime}-2 A \theta^{2} \sigma^{\prime}=\theta^{4}\left(A^{2} \sigma+B\right) \\
\frac{d \theta}{d t}=A \theta^{3}
\end{gathered}
$$

bağıntıları bulunur. Bu ifadelerden yararlanarak (3.15) denklemi yeniden düzenlenirse

$$
\frac{1}{2} f^{\prime \prime \prime}+f^{\prime} f^{\prime \prime}-\left(A^{2} w+B\right)
$$

elde edilir. Burada $f^{\prime}=P(w)$ olarak alınırsa yukarıda bulunan denklem

$$
\frac{1}{2} P^{\prime \prime}+P P^{\prime}-\left(A^{2} w+B\right)=0
$$

denklemine dönüşür, burada üç durum söz konusudur. Bunlar:

(i) $A=B=0$ olması durumu:

(3.25) denklemi $\frac{1}{2} P^{\prime \prime}+P P^{\prime}=0$ olur. Burada bir defa integral alınırsa,

$$
\frac{1}{2} P^{\prime}+\frac{1}{2} P^{2}+c_{2}=0
$$

denklemi elde edilir. Bu elde edilen denklemdeki $c_{2}$ keyfi sabiti için bazı özel durumlar göz önünde bulundurularak, denklemin rasyonel, trigonometrik ve hiperbolik çözümleri bulunabilir. Şöyle ki,

(3.26) denkleminde $c_{2}=0$ alınırsa,

çözümü elde edilir. (3.23) eşitliğinden

$$
P=\frac{1}{w}
$$

$$
\sigma^{\prime \prime}=0, \sigma^{\prime}=c_{1}, \sigma=c_{1} t+c_{0}
$$


olarak hesaplanır. Ayrıca (3.24) eşitliğinden

$$
\theta=\theta_{0}
$$

bulunur ve burada özel olarak

$$
\theta=\theta_{0}=1
$$

alınabilir. Bulunan bu (3.27) ve (3.28) sonuçlar (3.21) ve (3.22) eşitliklerinde yerlerine yazılırsa

$$
w=x+c_{1} t+c_{0} \text { ve } u_{0}=-c_{1}-1
$$

eşitlikleri bulunur. Bulunan bu ifadeler (3.13) dönüşümünde yerlerine yazılırsa verilen denklemin benzerlik çözümlerinden biri olan rasyonel çözüm fonksiyonu

elde edilmiş olur.

$$
u(x, t)=\frac{1}{\left(x+c_{1} t+c_{0}\right)}-c_{1}-1
$$

(3.26) denkleminde $c_{2}>0$ alınırsa,

özel olarak $c_{2}=\frac{1}{2}$ alındığında

$$
P=-\tan (w)
$$

bulunur. $w=x+c_{1} t+c_{0}$ eşitliği $P$ fonksiyonunda yerine yazılır ve $u_{0}=-c_{1}-1$ olduğu göz önüne alınır, bulunan ifade (3.13) dönüşümünde yerine konursa, denklemin benzerlik çözümlerinden olan trigonometrik çözüm fonksiyonu

olarak bulunur.

$$
u(x, t)=-\tan \left(x+c_{1} t+c_{0}\right)-c_{1}-1
$$

(3.26) denkleminde $c_{2}<0$ alınırsa,

özel olarak $c_{2}=-\frac{1}{2}$ alındığında

$$
P=\operatorname{coth}(w)
$$

bulunur. $w=x+c_{1} t+c_{0}$ olarak yukarıda yerine yazılır yerine yazılır ve $u_{0}=-c_{1}-1$ olduğu göz önüne alınır, bulunan ifade (3.13) dönüşümünde yerine konursa, denklemin benzerlik çözümlerinden bir diğeri olan

$$
u(x, t)=\operatorname{coth}\left(x+c_{1} t+c_{0}\right)-c_{1}-1
$$

hiperbolik çözüm fonksiyonu bulunmuş olur.

(i)A=0ve $B \neq 0$ olması durumu:

(3.23) eşitliğinde

$$
\sigma^{\prime \prime}=B, \sigma^{\prime}=B t+c_{1}, \sigma=\frac{1}{2} B t^{2}+c_{1} t+c_{0}
$$


olarak hesaplanır. Ayrıca (3.24) eşitliğinden

$$
\theta=\theta_{0}
$$

bulunur ve burada özel olarak

$$
\theta=\theta_{0}=1
$$

alınabilir. Bulunan sonuçlar (3.21) ve (3.22) eşitliklerinde yerlerine yazılırsa

$$
w=x+\frac{1}{2} B t^{2}+c_{1} t+c_{0} \text { ve } u_{0}=-B t-c_{1}-1
$$

bulunur. Bulunan bu ifadeler (3.13) dönüşümünde yerlerine yazılırsa

$$
u=P(w)-B t-c_{1}-1
$$

eşitliği elde edilir. Buradaki $P(w)$ fonksiyonu

denklemini sağlar.

$$
\frac{1}{2} P^{\prime \prime}+P P^{\prime}-B=0
$$

(ii) $A \neq 0$ ve $B=0$ olması durumu:

Genellik bozulmaksızın $A=-\frac{1}{2}$ alınabilir (3.24) eşitliğinden

$$
\frac{d \theta}{d t}=-\frac{1}{2} \theta^{3}
$$

elde edilir. Bu çözülürse $\theta=t^{-\frac{1}{2}}$ elde edilir. (3.14) eşitliğinde $\theta$ yerine $\theta=t^{-\frac{1}{2}}$ yazılırsa

$$
\sigma^{\prime \prime}+t^{-1} \sigma^{\prime}-\frac{1}{4} t^{-2} \sigma=0
$$

denklemi elde edilir ve bu denklem çözülürse

$$
\sigma=t^{-\frac{1}{2}}
$$

çözümü elde edilir. Bulunan bu değerler (3.21) ve (3.22) eşitliklerinde yerlerine yazılırsa

$$
w=\frac{x}{t^{\frac{1}{2}}}+\frac{1}{t^{\frac{1}{2}}} \text { ve } u_{0}=\frac{1}{2}\left(\frac{x}{t}+\frac{1}{t}\right)-1
$$

eşitlikleri bulunur. Bulunan bu değerler (3.13) dönüşümünde yazılırsa

$$
u=P(w) \frac{1}{t^{\frac{1}{2}}}+\frac{1}{2}\left(\frac{x}{t}+\frac{1}{t}\right)-1
$$

bulunur. Burada $P(w)$ fonksiyonu 


$$
\frac{1}{2} P^{\prime \prime}+P P^{\prime}+\frac{1}{4} w=0
$$

denklemini sağlar. Böylece verilen denklemin benzerlik çözümleri bulunmuş olur.

$\rightarrow$ (3.1) ile verilen Burgers like denklemi, Adomian ayrışım metodu ile çözelim. Verilen denklemin standart operatör oluşumu

$$
L_{t}(u)+u_{x}+N u+\frac{1}{2} L_{x x}(u)=0
$$

şeklinde ifade edilir. Burada, $N u=u u_{x}$ lineer olmayan terimi, $L_{t}=\frac{\partial}{\partial t}$ ve $L_{x x}=\frac{\partial^{2}}{\partial x^{2}}$ lineer diferansiyel operatörlerini ifade etmektedir. Burada, $L_{t}^{-1}$ ters operatörünün var olduğunu kabul edelim ve bunu $L_{t}^{-1}=\int_{0}^{t}() d$.$t şeklinde tanımlayalım. Bu ters operatörü (3.29) denklemine$ uygulanırsa

$$
L_{t}^{-1} L_{t}(u)=-L_{t}^{-1}\left(u_{x}\right)-L_{t}^{-1}(N u)-\frac{1}{2} L_{t}^{-1} L_{x x}(u)
$$

elde edilir. (3.30) eşitliğinden

$$
u(x, t)-u(x, 0)=-L_{t}^{-1}\left(u_{x}\right)-L_{t}^{-1}(N u)-\frac{1}{2} L_{t}^{-1} L_{x x}(u)
$$

yazılabilir. Burada $N u=u u_{x}=\sum_{n=0}^{\infty} A_{n}$ şeklinde ifade edilir ve $A_{n}$ Adomian polinomları da bir önceki bölümde olduğu gibi oluşturulur. $u_{0}$, bilindiğinden $n \geq 1$ olmak üzere $u_{n}(x, t)$ elemanları şu şekilde

$$
\begin{aligned}
& u_{1}=-L_{t}^{-1}\left(u_{0}\right)_{x}-L_{t}^{-1}\left(A_{0}\right)-\frac{1}{2} L_{t}^{-1} L_{x x}\left(u_{0}\right), \\
& u_{2}=-L_{t}^{-1}\left(u_{1}\right)_{x}-L_{t}^{-1}\left(A_{1}\right)-\frac{1}{2} L_{t}^{-1} L_{x x}\left(u_{1}\right), \\
& u_{3}=-L_{t}^{-1}\left(u_{2}\right)_{x}-L_{t}^{-1}\left(A_{2}\right)-\frac{1}{2} L_{t}^{-1} L_{x x}\left(u_{2}\right), \\
& \vdots \\
& u_{n}=-L_{t}^{-1}\left(u_{n-1}\right)_{x}-L_{t}^{-1}\left(A_{n-1}\right)-\frac{1}{2} L_{t}^{-1} L_{x x}\left(u_{n-1}\right), n \geq 1,
\end{aligned}
$$

yazılabilmektedir. $u_{0}=u(x, 0)$ verildiğine göre ayrışım serisinin diğer terimleri (3.32) yineleme formülü kullanılarak

$$
\begin{aligned}
& u_{0}=\frac{1}{2}+\frac{1}{2} \tanh \left(\frac{x}{2}\right), \\
& u_{1}=-L_{t}^{-1}\left(u_{0}\right)_{x}-L_{t}^{-1}\left(u_{0}\left(u_{0}\right)_{x}\right)-\frac{1}{2} L_{t}^{-1} L_{x x}\left(u_{0}\right)=-\frac{3}{8} \operatorname{tsech}^{2}\left(\frac{x}{2}\right) \\
& \left.u_{2}=-L_{t}^{-1}\left(u_{1}\right)_{x}-L_{t}^{-1}\left(u_{1}\left(u_{0}\right)_{x}+u_{0}\left(u_{1}\right)_{x}\right)-\frac{1}{2} L_{t}^{-1} L_{x x}\left(u_{1}\right)=-\frac{9}{32} t^{2} \operatorname{sech}^{2}\left(\frac{x}{2}\right) \tanh ^{-\frac{x}{2}}\right) \\
& u_{3}=-L_{t}^{-1}\left(u_{2}\right)_{x}-L_{t}^{-1}\left(u_{2}\left(u_{0}\right)_{x}+u_{1}\left(u_{1}\right)_{x}+u_{0}\left(u_{2}\right)_{x}\right)-\frac{1}{2} L_{t}^{-1} L_{x x}\left(u_{2}\right)=-\frac{9}{128} t^{3}(-2+ \\
& \cosh (x)) \operatorname{sech}^{4}\left(\frac{x}{2}\right)
\end{aligned}
$$

serinin dört terimi elde edilir. Bu elde edilen terimler (2.4.6) serisinde yerlerine yazılarak

$$
u(x, t)=\frac{1}{2}+\frac{1}{2} \tanh \left(\frac{x}{2}\right)-\frac{3}{8} \operatorname{tsech}^{2}\left(\frac{x}{2}\right)-\frac{9}{32} t^{2} \operatorname{sech}^{2}\left(\frac{x}{2}\right) \tanh \left(\frac{x}{2}\right)-\frac{9}{128} t^{3}(-2+
$$

$\cosh (x)) \operatorname{sech}^{4}\left(\frac{x}{2}\right)+\cdots$ 
ayrışım serisi oluşturulur ve yukarıdaki seri çözüm fonksiyonu kapalı fonksiyon olarak

$$
u(x, t)=\frac{1}{2}+\frac{1}{2} \tanh \left(\frac{1}{2} x-\frac{3}{4} t\right)
$$

şeklinde ifade edilir.

\section{SONUC}

$\mathrm{Bu}$ makalede, Burgers benzeri denklemin bazı seyahat eden dalga çözümlerini bulmak için Bäcklund Dönüşümü, Benzerlik indirgeme ve Adomian Ayrıştırma yöntemleri denkleme uygulanmıştır. Yukarıdaki yöntemlerin denkleme uygulanması sonucunda denklemin rasyonel, hiperbolik ve trigonometrik çözümleri elde edilmiştir. Daha sonra Mathematica 11.2 programını kullanarak bu çözümlerin denklemi sağladığı görülmüştür.

4. NOT

Bu makale İbrahim Enam İnan'ın doktora tezinin ilgili kısımlarından hazırlanmıştır.

\section{KAYNAKLAR}

[1] Y. Shang, Backlund transformation, Lax pairs and explicit exact solutions for the shallow water waves equation, Appl.Math.Comput. 187 (2007) 1286-1297.

[2] T.L. Bock, M.D. Kruskal, A two-parameter Miura transformation of the Benjamin-Ono equation, Phys. Lett. A 74 (1979) 173-176.

[3] V.B. Matveev, M.A. Salle, Darboux Transformations and Solitons, Springer, Berlin, 1991.

[4] A.M. Abourabia, M.M. El Horbaty, On solitary wave solutions for the two-dimensional nonlinear modified Kortweg-de Vries-Burger equation, Chaos Solitons Fractals 29 (2006) 354-364.

[5] W. Malfliet, Solitary wave solutions of nonlinear wave equations, Am. J. Phys. 60 (1992) 650-654.

[6] Y. Chuntao, A simple transformation for nonlinear waves, Phys. Lett. A 224 (1996) 77-84.

[7] F. Cariello, M. Tabor, Painleve expansions for nonintegrable evolution equations, Physica D 39 (1989) 77-94.

[8] M.L. Wang, Exact Solutions for a Compound KdV-Burgers equations, Phys. Lett. A 213 (1996) 279-287.

[9] E. Fan, Two new application of the homogeneous balance method, Phys. Lett. A 265 (2000) 353-357.

[10] G. Adomian, A Review of the Decomposition Method and some Recent Results for Nonlinear Equations, Math. Comp. Model., 13 (1990) 17-43.

[11] P.A. Clarkson, New similarity solutions for the modified boussinesq equation, J. Phys. A: Math. Gen. 22 (1989) 2355-2367.

[12] W. Malfliet, Solitary wave solutions of nonlinear wave equations, Am. J. Phys. 60 (1992) 650-654.

[13] E. Fan, Extended tanh-function method and its applications to nonlinear equations, Phys. Lett. A 277 (2000) 212218.

[14] S. A. Elwakil, S.K. El-labany, M.A. Zahran, R. Sabry, Modified extended tanh-function method for solving nonlinear partial differential equations, Phys. Lett. A 299 (2002) 179-188. 
[15] H. Chen, H. Zhang, New multiple soliton solutions to the general Burgers-Fisher equation and the KuramotoSivashinsky equation, Chaos Soliton Fract 19 (2004) 71-76.

[16] Z. Fu, S. Liu, Q. Zhao, New Jacobi elliptic function expansion and new periodic solutions of nonlinear wave equations, Phys. Lett. A 290 (2001) 72-76.

[17] S. Shen, Z. Pan, A note on the Jacobi elliptic function expansion method, Phys. Let. A 308 (2003) 143-148.

[18] H. T. Chen, Z. Hong-Qing, New double periodic and multiple soliton solutions of the generalized (2+1)dimensional Boussinesq equation, Chaos Soliton Fract 20 (2004) 765-769.

[19] Y. Chen, Q. Wang, B. Li, Jacobi elliptic function rational expansion method with symbolic computation to construct new doubly periodic solutions of nonlinear evolution equations, Z. Naturforsch. A 59 (2004) 529-536.

[20] Y. Chen, Z. Yan, The Weierstrass elliptic function expansion method and its applications in nonlinear wave equations, Chaos Soliton Fract 29 (2006) 948-964.

[21] M. Wang, X. Li, J. Zhang, The $\left(\frac{G}{G}\right)$-expansion method and travelling wave solutions of nonlinear evolutions equations in mathematical physics, Phys. Lett. A 372 (2008) 417-423.

[22] S.Guo, Y. Zhou, The extended $\left(\frac{G^{5}}{G}\right)$-expansion method and its applications to the Whitham-Broer-Kaup-like equations and coupled Hirota-Satsuma KdV equations, Appl.Math.Comput. 215 (2010) 3214-3221.

[23] H. L. Lü, X. Q. Liu, L. Niu, A generalized $\left(\frac{G^{\prime}}{G}\right)$-expansion method and its applications to nonlinear evolution equations, Appl. Math. Comput. 215 (2010) 3811-3816.

[24] L. Li, E. Li, M. Wang, The $\left(\frac{G^{F}}{G}, \frac{1}{G}\right)$ - expansion method and its application to travelling wave solutions of the Zakharov equations, Appl. Math-A J. Chin. U 25 (2010) 454-462.

[25] J. Manafian, Optical soliton solutions for Schrödinger type nonlinear evolution equations by the $\tan \left(\frac{\phi(q)}{2}\right)-$ expansion Method, Optik 127 (2016) 4222-4245.

[26] Mostafa M.A Khater, Emad H.M. Zahran, Soliton Soltuions of Nonlinear Evolutions Equation by using the Extended $\exp (-\varphi(\xi))$-expansion method, International Journal of Computer Applications, 145, 3 (2016) 1-5.

[27] J.H. He, Exp-function method for nonlinear wave equations, Chaos Solitons Fractals 30 (2006) 700-708

[28] Mostafa M.A Khater, "Extended $\exp (-\varphi(\xi))$-Expansion Method for Solving the Generalized Hirota-Satsuma Coupled KdV System", Global Journal of Science Frontier Research: F Mathematics and Decision Sciences, 15, 7, Version 1.0 Year 2015.

[29] Mostafa M.A Khater and Emad H.M. Zahran, "Modified extended tanh function method and its applications to the Bogoyavlenskii equation", Applied Mathematical Modelling,40, 1769-1775, 2016.

[30] İ.E.İnan. Kısmi Diferensiyel Denklemler için Bazı yaklaşım Metotları ve Uygulamaları. Doktora Tezi, Fırat Üniversitesi, Elazığ, Türkiye, 2004.

[31] G. Ebadi, A. Biswas, "Application of the $\left(\frac{G}{G}\right)$-expansion method for nonlinear diffusion equations with nonlinear source", Journal of the Franklin Institute, 347, 7, 1391-1398, 2010. 
[32] Zheyna Yan, "New explicit travelling wave solutions for two new integrable coupled nonlinear evolution equations", Physics Letters A, 292, 100-106, 2001.

[33] M.M.A. Khater, A.R. Seadawy and d. Lu, "Dispersive solitary wave solutions of new coupled Konno-Oono, Higgs field and Maccari equations and their applications", Journal of King Saud University, 30, 417-423, 2018.

[34] X. Zhao, L. Wang, W. Sun, "The repeated homogeneous balance method and its applications to nonlinear partial differential equations", Chaos Solitons and Fractals, 28, 448-453, 2006.

[35] A. Biswas, E. Topkara, S. Johnson, E. Zerrad, S. Konar, "Quasi-stationary optical solitons in non-Kerr law media with full nonlinearity”, Journal of Nonlinear Optical Physics \& Materials, 20, 309-325, 2011.

[36] A. Biswas, A.B. Aceves," Dynamics of solitons in optical fibers", Journal of Modern Optics, 48, 1135-1150, 2001.

[37] A.M. Wazwaz, "Burgers hierarchy: Multiple kink solutions and multiple singular kink solutions", Journal of the Franklin Institute, 347, 618-626, 2010.

[38] E.M.E. Zayed, M.A.M. Abdelaziz, "The two variables $\left(\frac{G^{*}}{G}, \frac{1}{G^{g}}\right)$-expansion method for solving the nonlinear KdVmKdV equation”, Mathematical Problems in Engineering, article ID 725061, 14 pp, 2012.

[39] Mostafa M.A Khater and Emad H.M. Zahran," New solitary wave solution of the generalized Hirota-Satsuma couple KdV system”, International Journal of Scientific \&Engineering Research, 6, 1324-1331, 2015.

[40] J. Manafian Heris, M. Lakestani, "Solitary wave and periodic wave solutions for variants of the KdV-Burger and the K(n, n)-Burger equations by the generalized tanh-coth method", Communications in Numerical Analysis, 1$18,2013$.

[41] A.M.Wazwaz, H. Triki," Multiple soliton solutions for the sixth-order Ramani equation and a coupled Ramani equation”, Applied Mathematics and Computation, 216, 332-336, 2010.

[42] A.M. Wazwaz, "The tanh-coth method for solitons and kink solutions for nonlinear parabolic equations”, Applied Mathematics and Computation, 188 (2), 1467-1475, 2007.

[43] J. Manafian Heris, I. Zamanpour," Analytical treatment of the coupled Higgs equation and the Maccari system via Exp-function method", Acta Universitatis Apulensis, 33, 203-216, 2013.

[44] A. Ergun, Süreksiz Difüzyon Operatörünün Çözümünün İntegral Temsili Jump Koşulları, Cumhuriyet Bilim Dergisi. 39 (1) (2018), 842-863.

[45] A. Ergun, R. Amirov, Kesintili difüzyon operatörü için Direkt ve Ters problemler noktaları, TWMS J. App. Müh. Matematik. 9 (1) (2019), 9-21.

[46] R. Amirov, A. Ergun, S. Durak, Yarı Ters Problemler Sturm-Liouville Denklemleri. Kismi Diferansiyel Denklemler için Sayısal Yöntemler. 37 (1) (2021), 915-924. 